\title{
CRDMAC: An Effective Circular RTR Directional MAC Protocol for Wireless Ad Hoc Networks
}

\author{
Huang Lu, Jie Li, and Zhongping Dong \\ Department of Computer Science \\ University of Tsukuba \\ Tsukuba Science City, Japan
}

\author{
Yusheng Ji \\ Information Systems Architecture Science Research Div. \\ National Institute of Informatics \\ Tokyo, Japan
}

\begin{abstract}
Directional antennas in wireless ad hoc networks (WANETs) offer great potential to reduce the radio interference, and improve the communication throughput. Using directional antennas, however, introduces a new problem in the wireless media access control (MAC), deafness, which may cause severe performance degradation. To solve the deafness problem, in this paper, we propose an effective Circular RTR Directional MAC (CRDMAC) protocol for WANETs by using a sub-transmission channel and RTR (Ready To Receive) packets, which modifies the IEEE 802.11 distributed coordinated function. The subchannel avoids collisions to other ongoing transmission and the RTR packets notify the neighbors that the mutual transmission has finished. The proposed MAC protocol decreases the binary exponential backoff time of the waiting nodes. We evaluate the CRDMAC protocol through simulations. Simulation results show that the proposed protocol outperforms the existing DMAC (directional MAC) protocol and the CRCM (Circular RTS and CTS MAC) protocol in terms of throughput and packet drop rate.
\end{abstract}

Keywords-WANETs, MAC protocol, deafness, circular directional antenna.

\section{INTRODUCTION}

A wireless ad hoc network (WANET) is a decentralized type of wireless network with a collection of wireless nodes. WANETs can dynamically self-organize into arbitrary or temporary network topologies without using any local infrastructure [1]. The main advantages of WANETs are flexibility, low cost, and robustness. WANETs can be easily set up, and can endure to natural catastrophes and wars. The characteristics of WANETs suite well for military activities, emergency operations, disaster recovery, and interactions $|2|$. The design of a WANET has to consider several interesting and difficult problems. For example, in the media access control (MAC) layer, the issues consist of the collision detection, the hidden and the exposed terminal problems, which demand new medium access algorithms [3]|4].

Traditionally, in WANETs, all nodes are equipped with omni-directional antennas, such an example is the IEEE 802.11 MAC protocol [5]. The MAC protocols with omnidirectional antennas waste a large portion of the network capacity. The usage of directional antennas has been proposed to alleviate this problem, in which antennas control the transmitting and receiving beams toward the receiver node only. Directional antennas have great potential to improve the network performance of WANETs, such as spatial reuse and range extension. Directional antennas can also provide higher gain and reduce interference by directing the radio beam towards a desired direction. |3|. Several MAC protocols using directional antennas for WANETs have been proposed recently, such as |6|, |7| and |8|, which are referred to as Directional MAC (DMAC) protocols. Most of the proposed protocols are adapting the usage of IEEE 802.11 over directional antennas, which is a standard and typical scheme for wireless LAN [9|| $10 \mid$.

The application of IEEE 802.11 to WANETs with directional antennas induces a problem of location-dependent carrier sensing, deafness. The deafness problem arises because of the beamforming in directional antenna operations [11], and may result in severe performance degradation by packet drop. Briefly, deafness is caused when a node attempting to initiate dialogs with a neighbor node, whereas the neighbor node is engaged in communication with another node.

In this paper, we propose a novel Circular RTR DMAC (CRDMAC) protocol for WANETs to solve deafness problem using directional antennas. The proposed protocol employs directional transmissions for the broadcasting of RTR (Ready To Receive) messages and the RTS (Request To Send) / CTS (Clear To Send) handshake, and increases the coverage area. It is based on a sub-transmission channel and circular directional RTR messages, which notice the neighbor nodes around the transmitter for the intended communication.

The rest parts of this paper are organized as follow. Section II provides an overview of related work. Section III provides the system description, and investigates the deafness problem and objectives. In section IV, we propose the novel circular RTR directional MAC protocol for WANETs. Section V analyzes the proposed CRDMAC protocol in network simulations. In the last section, we summarize the paper.

\section{RELATED WORK}

The usage of directional antennas has been studied in the context of both broadband and cellular networks over recent years. In this section, we present the related work including the preliminary knowledge for comprehending the CRDMAC protocol. 


\section{A. Multi-channel MAC}

In WANETs, the wireless medium is open and shared by nodes. Multiple nodes may try to access it simultaneously and get collision, if resource querying and acquiring are left uncontrolled. The media access control (MAC) protocol is to set the rules in order to enable efficient and fair sharing of the common wireless channel. The MAC protocol typically maximizes the channel utilization by having as many communications as possible $|10||12|$. Recently, the multi-radio multi-channel MAC protocols have been proposed in many researches, which use control separation techniques generally in multi-channel radio systems [13|[14]. Multi-channel diversity in MAC provides different purposes and usage: some intend to enable simultaneous data transmission, some reserve future data usage while the current data channel is occupied, some use separate signalling to solve hidden terminal problems. In this paper, we employ the multi-channel MAC protocol following the idea of splitting one radio channel into two subchannels, a data channel and a control channel, in purpose of alleviating deafness problem.

\section{B. IEEE 802.11 Distributed Coordinated Function}

IEEE 802.11 distributed coordinated function (DCF) is a contention based protocol of Carrier Sense Multiple Access with Collision Avoidance (CSMA/CA) and assumes the use of omni-directional antennas at the physical layer [5]. IEEE 802.11 DCF uses binary exponential backoff multiplicatively decrease the rate of transmission, in order to gradually find an acceptable rate. IEEE 802.11 is a CSMA/CA protocol which performs physical carrier sensing before initiating transmissions [10|. When the channel is sensed as idle for the DIFS (DCF Interframe Spacing) duration, the protocol invokes a backoff mechanism for contention resolution. However, IEEE 802.11 DCF wastes a large portion of the network capacity by reserving the wireless media.

\section{Classification of Directional Antennas}

Directional antennas are different from omni-directional antennas, which radiate and receive electromagnetic energy in all directions. They can be constructed to have certain preferential transmission and reception directions. There are two kinds of directional antennas, traditional directional antennas and smart antennas. Unlike traditional directional antennas, smart antennas consist of not only a number of radiating elements but also a network control unit. The control unit is normally implemented by using a digital signal processor, which is the intelligence of smart antennas. Smart antennas can be generally categorized as the following types |15||12|.

- Switched beam antenna: The antenna system uses a linear radio frequency network, which combines $M$ antenna elements to form $M$ predetermined directional beams. This antenna allows the selection of signals from the desired direction.

- Adaptive array antenna: Signals are processed adaptively by a combining network and summed to create a steerable radiation pattern. The Direction of Arrival (DoA) algorithm is applied for signal transmission/reception and continuous tracking.

In this paper, we utilize the switched beam antennas in the MAC protocol for WANETs.

\section{Circular DMAC Protocols}

Recently, many DMAC protocols have been proposed, which attempt to mitigate the issue of deafness. Korakis et al. [16] proposed a Circular RTS MAC, in which multiple RTS frames are sequentially transmitted on all beams for each data frame to scan the area around the transmitter. By maintaining a location table in each node, it can find the addressed receiver and deals with deafness and hidden terminal problems. The mechanisms offer significant improvement over that of omnidirectional transmissions, however, the excess control packets caused by circular directional RTS will degrade performance. Jakllari et al. [17] proposed a circular RTS and CTS MAC (CRCM) protocol, in which multiple directional CTS frames are transmitted consecutively in a circular way to handle deafness at the receiver side. Circular transmission, however, increasing the delay and incurs large control overhead. Gossain et al. |18| proposed a MAC protocol for directional antennas, in which multiple directional RTS and CTS messages are transmitted simultaneously through the antenna beams, which are in diametrically opposite directions with neighbors. In order to acquire the ongoing transmission information of neighbors, these schemes require circular transmission of RTS and CTS messages for each transmitted data packet, which may incur large control overhead.

\section{System Description And Problem Statement}

This section first presents the system description with the network structure and the antenna model taken into consideration. Then we investigate the deafness problem and objectives for WANETs with directional antennas.

\section{A. Network Structure}

We consider a WANET system consisting of a number of wireless nodes equipped with directional antennas, they are mobile and randomly distributed. In addition, we assume that, all the nodes use symmetric radio channel, which are homogenous in functionalities and capabilities.

An illustration of the network structure is shown in Figure 1 , where the nodes in the network are attempting for communications through MAC protocols. In this scenario, we can see that, nodes communicate or try to communicate with others in directional mode, node $C, X$ and $Y$ are enduring the deafness problem during the data transmission period between node $A$ and node $B$. We study this issue later in this section.

\section{B. Antenna Model}

In this paper, we consider that each node is equipped with a switched beam antenna which comprises $M$ fixed beam patterns, as shown in Figure 2. The antenna system possesses two separate modes, omni mode and directional mode. In omni mode, a node receives signals from all directions with gain 


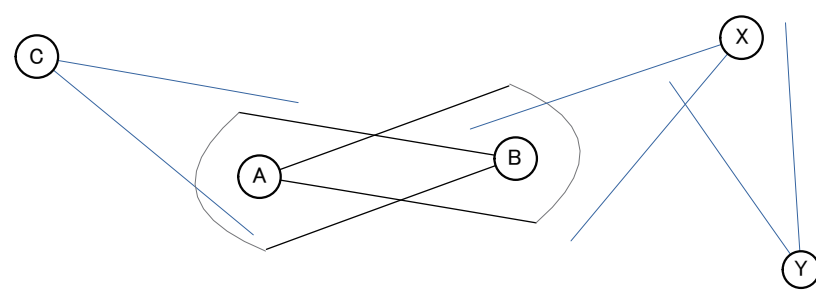

Fig. 1. An illustration of the network structure with deafness problem

$G^{o}$. A node in idle state waits for signals in omni mode. When a signal is sensed in omni mode, the antenna locates the direction on which the signal power is strongest and goes into the directional mode. In the directional mode, a node points its beam toward a specific direction with gain $G^{d}$ $\left(G^{d}>G^{o}\right)$. Most existing research utilizes the same antenna model. In the CRDMAC protocol, the omni mode is utilized for receiving signals, whereas the directional mode is utilized for data transmission and reception. We assume that the pattern in the directional mode is a perfect circular sector with a constant gain in the sector, such that, there is no antenna gain outside the sector.

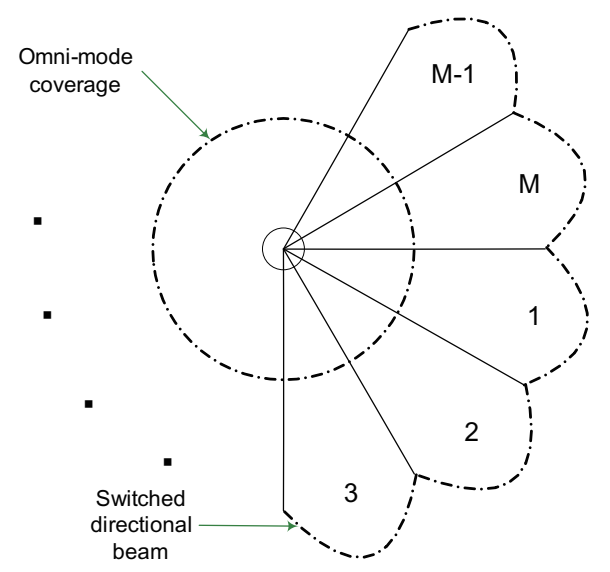

Fig. 2. Antenna model with $\mathrm{M}$ beams

In the directional mode, the antenna can transmit a signal in any direction, using an array of elements. Individual omnidirectional transmissions from these elements interfere with each other, resulting in the increase of the signal strength in one or more directions, and the elimination in other directions. There are directional antennas with 1 (omni-directional) 2, 4, 8,16 etc. elements [3]|19|. As the number of antenna elements increases, the beam width and the signal gain can be controlled more effectively. An important element of the CRDMAC protocol covers the whole area around the transmitter with successive sequential transmission. In the rest of the paper, we assume that it can provide effective omni transmission with $M$ sequential directional transmissions when we have $M$ antenna elements.

\section{Deafness Problem}

We explain the deafness problem using the scenario in Figure 1. Assume that all nodes are idle and that node $B$ intends to transmit a data packet to node $A$. DMAC requires node $B$ to beamform in the direction of node $A$, and detect if the channel is idle for a DIFS duration. When the channel is found idle, node $B$ proceeds to the backoff phase and counts down the backoff counter while still beamformed towards node $A$. Observe that while node $B$ is counting down its backoff counter, node $X$ may intend to communicate with node $B$. If node $X$ completes its own backoff before node $B$ and transmits an RTS to node $B$, node $B$ would not receive the RTS. In the absence of a reply from node $B$, node $X$ would repeatedly backoff and retransmit the RTS, until the dialog between node $B$ and node $A$ is over. Unproductive retransmissions are the outcome of deafness.

Now consider the case where node $B$ has multiple packets to transmit to node $A$. Once node $B$ has finished transmitting the first packet, it immediately prepares for transmitting the next packet by beamforming in the direction towards node $A$, and then repeating the sequence of DMAC operations, i.e., carrier sense, backoff, RTS, CTS, etc. Notice that, unless node $B$ is in the omni mode, node $X$ would not be able to communicate with node $B$. When using DMAC, node $B$ would not switch to the omni mode until it has finished transmitting all its queued packets for node $A$. If node $B$ remains backlogged for a long time, node $X$ may end up dropping the packets meant for node $B$. As well as, repeated failure to communicate to node $B$, causes node $X$ 's contention window (CW) to grow exponentially. If node $X$ has multiple packets queued for node $B$, it would remain engaged either in backoff or in transmitting a directional RTS. Node $Y$ would experience prolonged deafness, until $\mathrm{X}$ has dropped all its packets. When the nodes in a chain transmission, where none of the nodes communicates successfully, evokes a new problem, a "deadlock" [11]. It is a serious problem on multihop transmission, when the intended receiver of a node is a transmitter.

\section{Objectives}

The deafness problem and the deadlock problem caused by it waste the network capacity and result in unfairness as well. When multiple nodes attempt to communicate with the same node, the node that wins channel contention retains the privilege to access the channel for a long time. Although the receiver remains busy almost all the time, the other transmitter nodes experience unfairness.

In this paper, we focus on deafness and the deadlock problem from the conditions above. We propose a novel CRDMAC protocol to solve this problem.

\section{PRoposed CRDMAC PRotocol FOR WANETS}

This section presents the details of proposed CRDMAC protocol, including the antenna gain employed in the protocol.

\section{A. The Gain of Antennas}

The antenna radiates the time-averaged power in all directions. The gain of an antenna can be defined as

$$
G(\theta, \phi)=\eta \frac{U(\theta, \phi)}{U_{a \nu e}},
$$




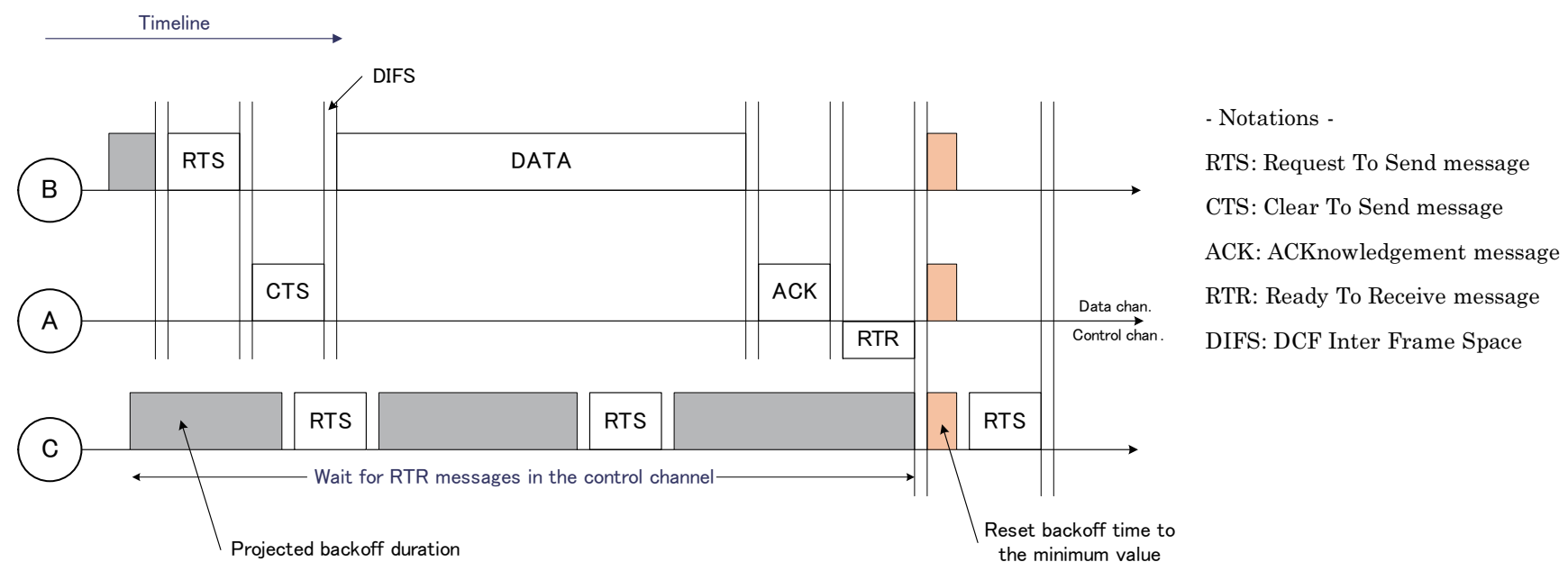

Fig. 3. An illustration of the operation of CRDMAC

where $U(\theta, \phi)$ is the power density in direction $(\theta, \phi), U_{\text {ave }}$ is the average power density over all directions and $\eta$ is the efficiency of the antenna. If the antenna transmits power equally in all directions, then $U(\theta, \phi)$ will be equal to $U_{a \nu e}$ and the antenna is called isotropic. An isotropic antenna has a spherical pattern and is only used for analytical purpose.

The power gain $G(\theta, \phi)$ of an antenna is the ratio of radiation intensity to average intensity over all directions. If no direction is specified, the gain usually means the maximum gain value over all directions. Due to the reciprocity, all the gain and radiation pattern characteristics are known to be the same for both transmission and reception.

\section{B. Operation of CRDMAC Protocol}

The key contribution of Circular RTR DMAC (CRDMAC) protocol lies in the use of two sub-channels and circular transmission of RTR (Ready To Receive) to alleviate deafness. We observed that deafness occurs primarily because a transmitter is unaware of the activities of its intended receiver. The main idea of the CRDMAC protocol is to inform a node's neighborhood of its activity.

In CRDMAC, the common channel is split into two subchannels: a data channel and a narrow control channel. We adopt the control separation concepts from |13| for transmission in multi-channel MAC protocol. RTS, CTS, DATA and ACK packets are transmitted on the data channel. The RTR messages are assigned on the control channel. We assume that an idle transceiver is capable of tuning into both data and control channels.

The RTR packets serve as indicators that nodes $B$ and $A$ were recently engaged in communication. A neighboring node, $C$, unable to communicate with node $A$ or node $B$ in the meanwhile, can get the RTR packets in the control channel, as an indication of recent deafness. The information in RTR packets is only the ID of sender so the control narrow can be very narrow. The neighbors who received it can know which neighbor was transmitting just now by the ID of RTR packets.

After exchanging the DATA/ACK, both the sender $A$ and the receiver $B$ will turn to control channel. Then circularly send RTR packets to notify its neighbors that it has finished its transmission which maybe has caused deafness. Because the RTR packets may interfere with the transmission between other neighbors we send RTR in control channel. The time to send a RTR packet is

$$
t_{r t r}=\frac{S_{\text {control }}}{R_{\text {control }}}+\iota+c,
$$

where $S_{\text {control }}$ is the size of the RTR packet, $R_{\text {control }}$ is the data rate of control channel, $\iota$ the channel latency and $c$ the beam switching time.

We illustrate the operation of CRDMAC protocol in Figure 3 , where node $B$ is transmitting data to node $A$, meanwhile, node $C$ attempts to communicate with node $A$. However, node $A$ is deaf to node $C$, thus node $C$ waits for RTR messages in the control channel. We introduce the operation of proposed CRDMAC protocol with both transmitting sides and waiting sides taken into account.

1) Transmitting sides: In this scenario, node $B$ and node $A$ are the transmitting sides, in this case, node $B$ initiates dialogs with node $A$ and transmits data to node $A$.

- Step 1. Node $B$ initiates the RTS message, and broadcast it to node $A$.

- Step 2. After received the RTS message, node $A$ replys node $B$ the CTS in a Four-Way Handshake [5] on data channel.

- Step 3. After received the CTS message, node $B$ transmits data to node $A$ and get the ACK back if succeeded.

- Step 4. When the Four-Way Handshake of data communication is over, node $B$ tunes into the control channel for declarations. It initiates RTR messages, then transmits circularly with the directional antenna to their neighbors. As we have mentioned in section III, we adapt antennas with predefined number of beams, $M$ in Figure 2, which cover the whole area around the transmitter. Therefore, node $B$ starts transmitting its RTR in a predefined direction, assuming with beam 1 , then turns its transmission 
beam sending the same RTR with beam 2 . It continues this procedure repeatedly (until beam $\mathrm{M}$ ) until the transmission of RTR covers all the area around the transmitter.

- Step 5. After sending the RTR packets circularly, node $B$ tunes back to the data channel for other operations, e.g., starting communication with another node.

2) Waiting sides: In this scenario, node $C$ is the waiting side, and it has to wait for RTR messages since node $A$ is deaf to it.

- Step 1. Since node $C$ is attempting transmission towards node $A$, it sends RTS packet to node $A$.

- Step 2. Node $C$ waits for a backoff time and tunes to control channel for RTR messages, because no CTS replying from node $A$. When the backoff counter reaches 0 , node $C$ tunes to the data channel, transmits the RTS packets again, and waits for a backoff time repeatedly if no CTS received.

- Step 3. If node $C$ receives a RTR packet, it reset the backoff time to the minimum value $C W_{\min }$, and broadcasts the RTS packet to node $A$ for potential communication.

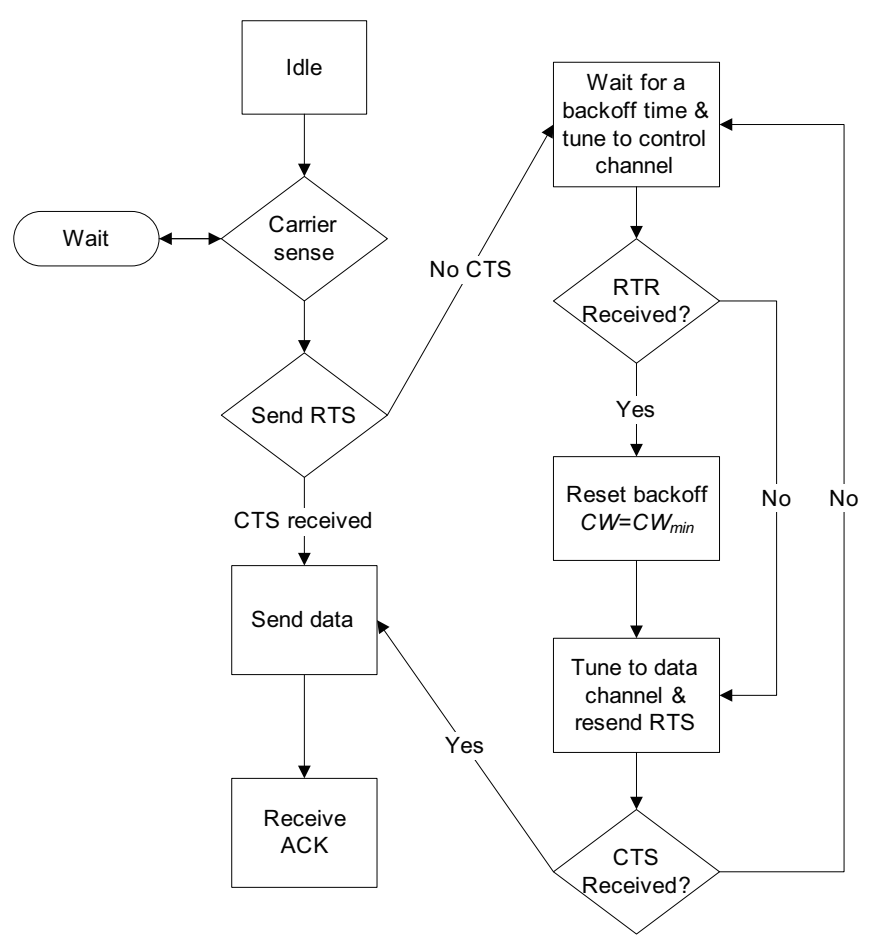

Fig. 4. Flowchart of the waiting node

Figures 4 shows the flowchart of the proposed CRDMAC protocol at the waiting sides (node $C$ ).

Observing in Figure 3, the projected backoff duration shows the duration over deafness, where node $C$ may continue backing off in the absence of the RTR packet. In that case, node $B$ will have won channel contention yet again. However, in the proposed CRDMAC protocol, node $C$ can compete against node $B$ fairly.

\section{Performance Evaluation}

In this section, we focus on the throughput and the packet drop rate from the network simulation. We use the network simulator QualNet $4.5[20 \mid$ with Java Runtime Environment 6 [21] to simulate the proposed CRDMAC protocol.

\section{A. System Parameters and Settings}

In the network simulation experiments, we study two typical scenarios below to do simulations. Scenario 1 indicates the random deployed topology condition when the deafness problem happens. Scenario 2 indicates the common grid topology condition.

We assume that, the physical channel is error free and the propagation delay is zero, which bandwidth is splited between two subchannels as a data channel and a control channel. The transmission packet length is set as constantly equal to 1024 bytes. the transmission range of directional antenna is $R_{d}=300 \mathrm{~m}$, the number of beam for a circule directional antenna is 4 beams, and the switching time of one beam is $c=1.1 \mathrm{~ms}$. The channel latency is set to $\iota=3.1 \times 10^{-3} \mathrm{~ms}$, and the slot time of DIFS is $t=10 \mu \mathrm{s}$. The minimum value of node contention window $(\mathrm{CW})$ is $C W_{\min }=15 \mathrm{~ms}$, as the $\mathrm{CW}$ exponentially grows, the maximum value of $\mathrm{CW}$ is $C W_{\max }=130 \mathrm{~ms}$. We compare the proposed CRDMAC protocol with DMAC protocol [6] and CRCM protocol |17].

We evaluate the protocols with two performance metrics, throughput and packet drop rate. Network throughput refers to the average data rate of successful data or message delivery over a communication link. Packet drop rate is a useful measure for WANETs, which is relative to the data flows. We are trying to determine how much we alleviate the deafness problem by decreasing the drop rate and increasing the throughput.

\section{B. Scenario 1}

We evaluate the performance of the scenario demonstrated in Figure 5. In this experiment, node $C$ wants to transmit to node $A$ while node $B$ is transmitting to node $A$, even when node $B$ has finished the transmission, node $B$ will transmit to node $A$ again and again. Node $A$ cannot hear node $C$ and will not reply CTS to node $C$. As we have discussed in the previous sessions, the backoff time of node $C$ will be set longer after times, which leads to deafness problem.

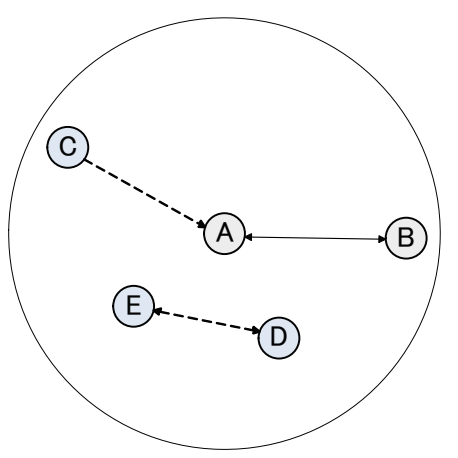

Fig. 5. Scenario 1 for CRDMAC protocol simulation 
We first consider the packet drop rate. Because the existing DMAC protocol cannot alleviate the deafness problem, node $C$ must wait for its binary exponential backoff time, even when node $A$ has finished its transmission with node $B$. In DMAC protocol, the probability of dropping packet of node $C$ is very high, because node $C$ conflicts with node $B$ for many times. But the CRDMAC protocol solves the deafness problem, where node $C$ can compete with node $B$ fairly. It does not drop its packets as we see in Table I

TABLE I

THE COMPARISON OF DROP RATE

\begin{tabular}{|c|c|c|c|}
\hline & CRDMAC & CRCM & DMAC \\
\hline Packet drop rate of node $C$ & 0 & 0 & $40 \%$ \\
\hline
\end{tabular}

Now we compare the proposed CRDMAC protocol with DMAC protocol in term of throughput to verify the effectiveness. Simulation results are the average of 100 runs. The result in Figure 6 shows that, the CRDMAC protocol performs better than DMAC protocol because it solves deafness properly. As node $A$ informs node $C$ for the intended transmissions by the circular RTR, it induces a higher throughput than that of DMAC protocol. As Node $C$ receives the RTR packets and set its backoff time to the minimum value, node $C$ can compete with node $B$ fairly and will not drop its packets for the long time waiting.

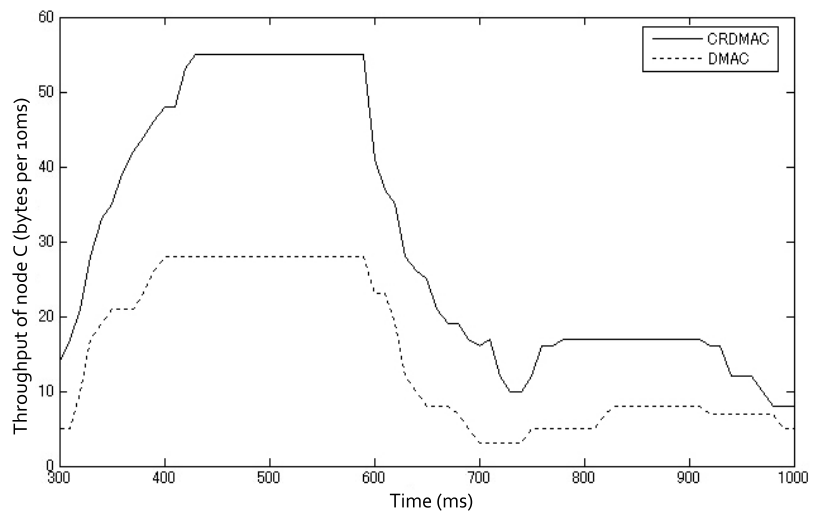

Fig. 6. Throughput of node $C$ in scenario 1

Figure 7 shows the network throughput of CRDMAC protocol compared with CRCM protocol. The result shows that, the network throughput of CRDMAC protocol is better than that of CRCM protocol, because we use the control channel to send RTR packets which don not interfere with the transmission of node $D$ and node $E$. In CRCM, the circular RTS and CTS will be sent to its neighbors when taking handshake and the transmission of node $D$ and node $E$ will be interfered.

\section{Scenario 2}

The second simulation uses the scenario in figure 8 . There are 9 nodes in a grid topology. The neighbors of a node can be concluded by the circular range around node 5, that depicts the coverage range of the 4 beams of this position, thus each

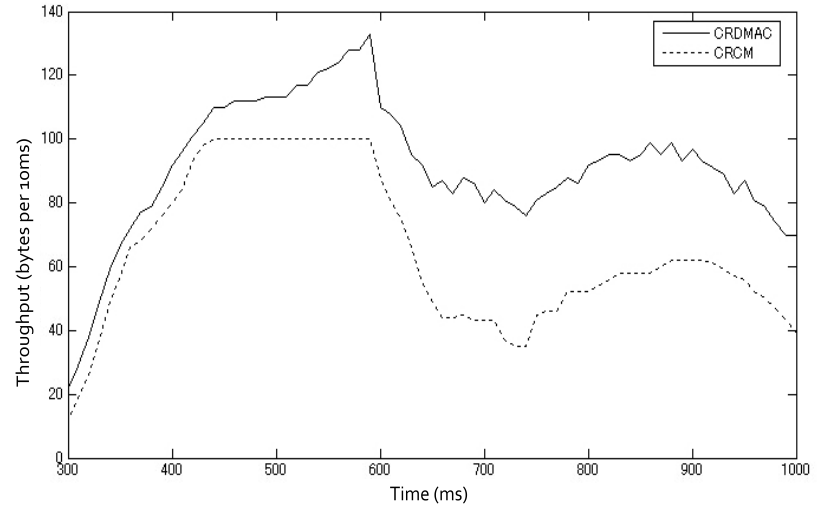

Fig. 7. Network throughput in scenario 1

node only can communicate nodes in horizontal and vertical directions. During the network simulation, we randomly let two nodes communicate with each other at one time frame, and change the transmission rate of the sending node.

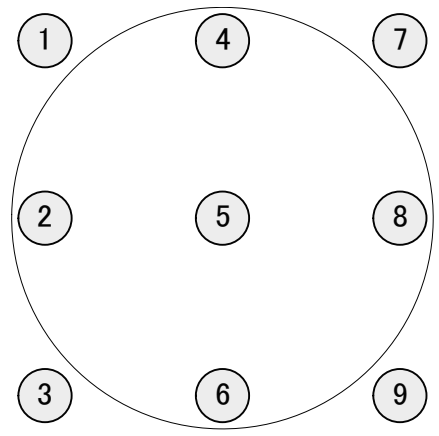

Fig. 8. Scenario 2 for CRDMAC protocol simulation

The simulation results are shown in Figure 9. In the low transmission rate, the CRDMAC protocol has lower throughput than that of DMAC protocol. This happens due that, the available data rate for communication has to allocate part of the bandwidth to the control channel. CRDMAC protocol is inferior to DMAC protocol because of the overhead of circular transmissions, however, as the sending rate increases, this extra overhead is quickly canceled.

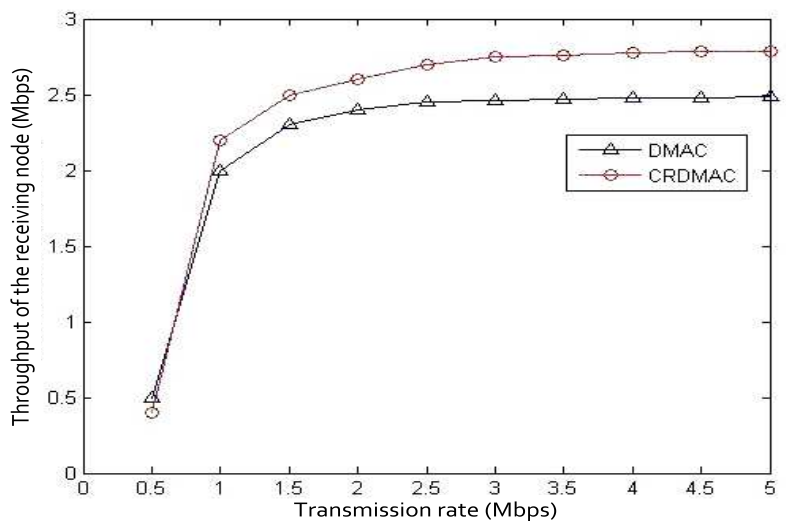

Fig. 9. Throughput of the receiving node in scenario 2 


\section{CONCLUSion AND Future Work}

In this paper, we have proposed a Circular RTR DMAC (CRDMAC) protocol, which use a novel receiver-initiated mechanism to handle the deafness problem. We use circular the RTR message to handle deafness reactively, and alleviate the deafness problem in WANETs using circular directional antennas. The transmission time of circular messages will not delay data transmission, since the circular messages are sent after the data transmission. The circular packets will not interfere with the neighbors by using a sub-channel which is better than CRCM. In the future work, we plan to enhance the CRDMAC protocol by simulations with various parameters and incorporate with QoS requirements.

\section{ACKNOWLEDGMENTS}

This work has been partially supported by Grant-in-Aid for Scientific Research of Japan Society for Promotion of Science (JSPS).

\section{REFERENCES}

[1] C. K. Toh, Ad Hoc Mobile Wireless Networks: Protocols and Systems. Prentice Hall; 1 edition, 2002.

[2] M. Rubinstein, I. Moraes, M. Campista, L. Costa, and O. Duarte, "A Survey on Wireless Ad Hoc Networks," in Mobile and Wireless Communication Networks, IFIP International Federation for Information Processing, 2006, vol. 211, pp. 1-33.

[3] H. Dai, K.-W. Ng, and M.-Y. Wu, "An Overview of MAC Protocols with Directional Antennas in Wireless ad hoc Networks," in Proc. of Second International Conference on Wireless and Mobile Communications, ICWMC'06, 2006.

[4] R. Ramanathan, J. Redi, C. Santivanez, D. Wiggins, and S. Polit, "Ad hoc networking with directional antennas: a complete system solution," IEEE Journal on Selected Areas in Communications, vol. 23, no. 3, pp. 496-506, 2005.

[5] IEEE 802.11, Wireless LAN medium access control (MAC) and physical layer (PHY) specifications, IEEE Standard, 1999.

[6] Y.-B. Ko, V. Shankarkumar, and N. H. Vaidya, "Medium access control protocols using directional antennas in ad hoc networks," in Proc. of IEEE 9th Annual Joint Conference of the IEEE Computer and Communications Societies, INFOCOM'00, 2000.

[7] R. R. Choudhury, X. Yang, R. Ramanathan, and N. H. Vaidya, "Using directional antennas for medium access control in ad hoc networks," in Proc. of the 8th annual international conference on Mobile computing and networking, MobiCom'02, 2002.

[8] M. Takai, J. Martin, R. Bagrodia, and A. Ren, "Directional virtual carrier sensing for directional antennas in mobile ad hoc networks," in Proc. of the 3rd ACM international symposium on Mobile ad hoc networking \& computing, MobiHoc'02, 2002.

[9] S. Kumar, V. S. Raghavan, and J. Deng, "Medium Access Control protocols for ad hoc wireless networks: A survey," Ad Hoc Networks, vol. 4, no. 3, pp. 326-358, 2006.

[10] R. Jurdak, C. Lopes, and P. Baldi, "A survey, classification and comparative analysis of medium access control protocols for ad hoc networks," IEEE Communications Surveys \& Tutorials, vol. 6, no. 1, pp. 2-16, 2004.

[11] R. Choudhury and N. H. Vaidya, "Deafness: a MAC problem in ad hoc networks when using directional antennas," in Proc. of the 12th IEEE International Conference on Network Protocols, ICN2004, 2004.

[12] O. Bazan and M. Jaseemuddin, "A Survey On MAC Protocols for Wireless Adhoc Networks with Beamforming Antennas," IEEE Communications Surveys \& Tutorials, vol. PP, no. 99, pp. 1-24, 2011.

[13] J. So and N. H. Vaidya, "Multi-channel mac for ad hoc networks: handling multi-channel hidden terminals using a single transceiver," in Proc. of the 5th ACM international symposium on Mobile ad hoc networking and computing, MobiHoc'04, 2004.
[14] J.-P. Wang, M. Abolhasan, F. Safaei, and D. Franklin, "A survey on control separation techniques in multi-radio multi-channel MAC protocols," in Proc. of International Symposium on Communications and Information Technologies, ISCIT'07, 2007.

[15] P. H. Lehne and M. Pettersen, "An overview of smart antenna technology for mobile communications systems," IEEE Communications Surveys \& Tutorials, vol. 2, pp. 2-13, 1999.

[16] T. Korakis, G. Jakllari, and L. Tassiulas, "CDR-MAC: A Protocol for Full Exploitation of Directional Antennas in Ad Hoc Wireless Networks," IEEE Transactions on Mobile Computing, vol. 7, no. 2, pp. $145-155,2008$

[17] G. Jakllari, I. Broustis, T. Korakis, S. Krishnamurthy, and L. Tassiulas, "Handling asymmetry in gain in directional antenna equipped ad hoc networks," in Proc. of IEEE 16th International Symposium on Personal, Indoor and Mobile Radio Communications, PIMRC'05, 2005.

[18] H. Gossain, C. Cordeiro, and D. Agrawal, "MDA: an efficient directional MAC scheme for wireless ad hoc networks," in Proc. of IEEE Global Telecommunications Conference, GLOBECOM'05, 2005.

[19] M. Takata, M. Bandai, and T. Watanabe, "Performance Analysis of a Directional MAC Protocol for Location Information Staleness in MANETs," IPSJ Digital Courier, vol. 1, pp. 518-527, 2005.

[20] "QualNet simulator," Scalable Network Technologies. Available: http:// www.scalable-networks.com/

[21] "Java Runtime Environment," Java, Sun Microsystems, Oracle Corporation. Available: http://http://www.java.com/ 